\title{
Abrupt vs. gradual destabilization at copying affects the subsequent evolution of graphic patterns accuracy at reproduction.
}

\author{
V. Kostrubiec* $\quad$ P.G. Zanone* \\ (*) Université de Toulouse, UPS, PRISSMH, \\ 118 route de Narbonne, F-31062 Toulouse Cedex 9, France \\ E-mail:kostrubi@,cict.fr; zanone@,cict.fr
}

\begin{abstract}
Participants should trace graphic patterns with respect to a visual model (copying) and, after an interval of varying length $(0,10$ and $20 \mathrm{~s})$, reproduce the patterns from memory (reproduction). The $0^{\circ}, 45^{\circ}$, $90^{\circ}, 135^{\circ}$ and $180^{\circ}$ relative phase patterns were analyzed. At copying, the patterns were destabilized by manipulating movement frequency. The results revealed that absolute error and variability were higher when participants traced the patterns at a rapid rather than at a spontaneous movement frequency. Later, the effect of destabilization decayed resulting in accuracy improvement. After an abrupt change from rapid to spontaneous movement frequency at copying, the improvement in accuracy was manifested with the 0 $s$ interval. After a gradual decrease in frequency, the improvement in accuracy appeared for the 10 and $20 \mathrm{~s}$ interval. The results indicate that the evolution of accuracy at reproduction was sensitive to how the destabilization was administered at copying.
\end{abstract}

\section{Introduction}

Recently, some authors [1] suggested that the evolution of performance at recall depends on how environmental information specific to the task, changed over time during practice. Basically, the task is recalled well when the environmental information changed gradually and it is forgotten when the context changed abruptly. In the same vein, we shall test here the effect of abrupt vs. gradual changes in the stability of graphic patterns on their later reproduction from memory.

Coordination patterns can be destabilized by increasing movement frequency [2-3]. When this frequency constraint is removed, the patterns recover stability and accuracy over time, as if - so to say - the motor system was forgetting the destabilization effect. Our main hypothesis is that the performance of coordination patterns that have previously undergone a gradual change in stability improves, whereas the performance of patterns whose stability changed abruptly does not.

In the present experiment each trial was composed of three periods: copying, interval, and reproduction, reminiscent of the three stages in the classic short-term memory paradigm: encoding, retention interval and recall. At copying, participant should perform a graphic pattern with respect to a visual model. Patterns had to be produced at spontaneous, at highest and at gradually decreasing movement frequency. Then, the visual model was removed, and after an interval of various duration, the pattern was to be reproduced from memory. We expect that after copying at gradually decreasing movement frequency, the accuracy at reproduction should improve most.

The production of graphic patterns such as ellipses of different eccentricities can be characterized by typical values of relative phase [4-5]. The $0^{\circ}$ pattern give rise to a right-slanted line, $180^{\circ}$ to a left-slanted line, $90^{\circ}$ to a circle, $45^{\circ}$ to right-slanted ellipse and $135^{\circ}$ to left-slanted ellipse. When thirteen ellipsoids, characterized by a relative phase ranging from $0^{\circ}$ to $180^{\circ}$ by steps of $15^{\circ}$, were used as visual models, only five graphic patterns were produced with accuracy and stability, biasing the production of the neighboring ones: $0^{\circ}, 45^{\circ}, 90^{\circ}, 135^{\circ}$ and $180^{\circ}$. We shall focus on these five particular graphic patterns to test the effect of destabilization during copying on their subsequent reproduction.

\section{Method}

\subsection{Participants}

Twelve naïve, unpaid volunteers, 4 male and 8 female, aged about 23 , took part in the study. The study was approved by the local ethical committee of Paul Sabatier University and participants provided a written

This is an Open Access article distributed under the terms of the Creative Commons Attribution-Noncommercial License 3.0, which permits unrestricted use, distribution, and reproduction in any noncommercial medium, provided the original work is properly cited. 
informed consent in accordance with the Helsinki Declaration.

\subsection{Material and apparatus}

The graphic task was performed on a computercontrolled WACOM (Intuos 2) (DTF 720) $x-y$ digitizing tablet, using an electronic pen. As soon as the electronic pen touched the tablet, the $\mathrm{x}$ and $\mathrm{y}$ spatial coordinates of the performed trajectories were digitized at $100 \mathrm{~Hz}$, and stored with an accuracy of $0.2 \mathrm{~mm}$ for further analysis.

Thirteen black ellipsoids characterized by relative phases ranging from $0^{\circ}$ to $180^{\circ}$ by steps of $15^{\circ}$ were used as visual models. These patterns were displayed at random, in the centre of the white screen of the digitized tablet for $20 \mathrm{sec}$ (Figure 1, left panels). Four auditory tones, of 1040, 840, 640 and $440 \mathrm{~Hz}$ and of $0.1 \mathrm{sec}$ duration were used. The highest tone was displayed at the beginning of each trial. The three lower tones were used in gradual copying condition at 5, 10 and $15 \mathrm{sec}$ of the display (Figure 2, bottom left panel). The lowest tone was presented at $15 \mathrm{sec}$ of the display in rapid copying condition (Figure 2, top left panel).

A white screen was presented during the interval between copying and reproduction and a white screen with the writing zone during the reproduction (Figure 2 , central and right panels). The procedure and the visual display were controlled through the Matlab $\mathbb{C}$ Psychophysics Toolbox on a customized $3 \mathrm{GHz}$ PC.

\subsection{Procedure}

Participants were instructed to trace in superposition of the visual model using counter-clockwise direction of pen tip rotation. At each trial, copying lasted $20 \mathrm{~s}$ and reproduction $10 \mathrm{~s}$. The interval between copying and reproduction was manipulated and lasted 0,10 , or $20 \mathrm{~s}$. Movement frequency was manipulated by verbal instructions. Participants were asked either to trace the figures at frequency they felt most comfortable (spontaneous) or to trace them as fast as possible (rapid). When movement frequency was required to change, an auditory signal was outputted. Three copying conditions were compared: 1) spontaneous copying: participants were instructed to trace the pattern at spontaneous movement frequency during the whole copying, 2) rapid copying: they should trace the pattern at the highest movement frequency for $15 \mathrm{~s}$ and at a spontaneous movement frequency for the last $5 \mathrm{~s}$; and 3) gradual copying: the movement frequency should be decreased from the highest to the spontaneous frequency by four plateaus of $5 \mathrm{~s}$.
After familiarization, an experimental session began using the thirteen experimental ellipses for each of the nine experimental conditions (3 Copying $\times 3$ Interval). The conditions were presented at random and the whole experiment lasted about $2 \mathrm{~h}$.

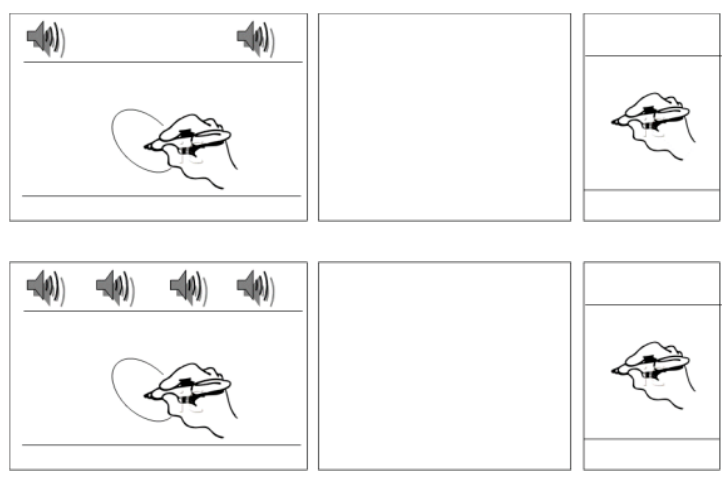

Fig. 1. Experimental design for rapid (top panel) and for gradual (bottom panel) copying conditions (see text for details).

\subsection{Data Analysis}

For the sake of brevity, data analysis is focused here on five patterns only $\left(0^{\circ}, 45^{\circ}, 90^{\circ}, 135^{\circ}, 180^{\circ}\right)$. Copying conditions were treated in 4 consecutive periods of $5 \mathrm{~s}$. For each copying Period and for each reproduction stage, the number of cycles, the point-estimated RP between $\mathrm{x}$ and $\mathrm{y}$ spatial coordinates were measured. The RP were averaged and the corresponding index of variability computed using circular statistics. Note that circular index of variability, $\mathrm{R}$, decreases from 1 to 0 as variability increases. For this raison, it will be coined as stability index henceforth. The accuracy of the produced patterns was assessed through the absolute error (AE), that is, the absolute value of the difference between the produced and the required PR.

An Improvement Index was calculated as the difference between AE produced at copying and the $\mathrm{AE}$ produced at reproduction. A positive index indicates an increase in accuracy between copying and reproduction. A negative index indicates a decrease in accuracy between the two stages.

All experimental factors were treated as repeated measures. If the data did not satisfy the sphericity of variances assumption with regard to GeisserGreenhouse's index, the required adjustments were applied to the degrees of freedom for the F-tests. The analysis was complemented by polynomial contrasts to test whether the distribution of data presents a reliable linear trend, and by t-tests with Bonferroni correction for multiple comparisons. 


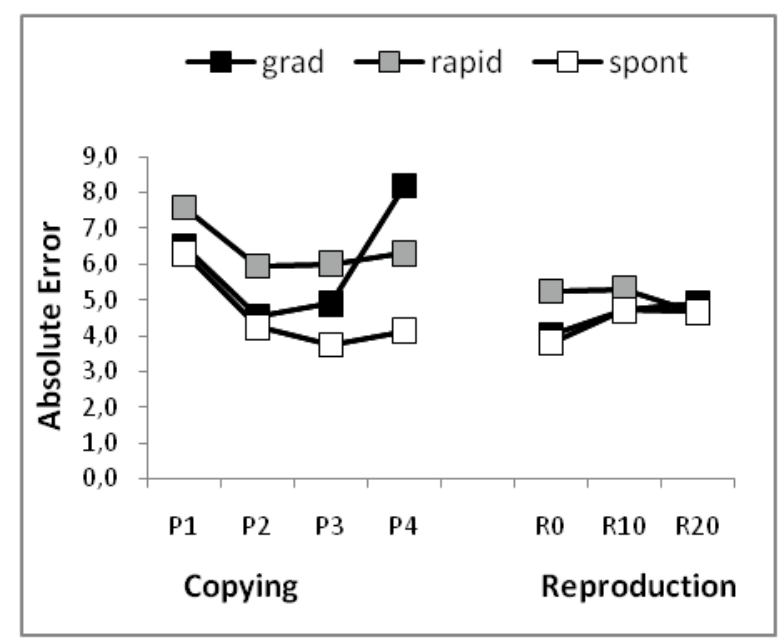

Fig. 2. On the left: Absolute Error at Copying as a function of copying Period and of copying condition. On the right: Absolute Error at Reproduction as a function of Interval and of copying condition.

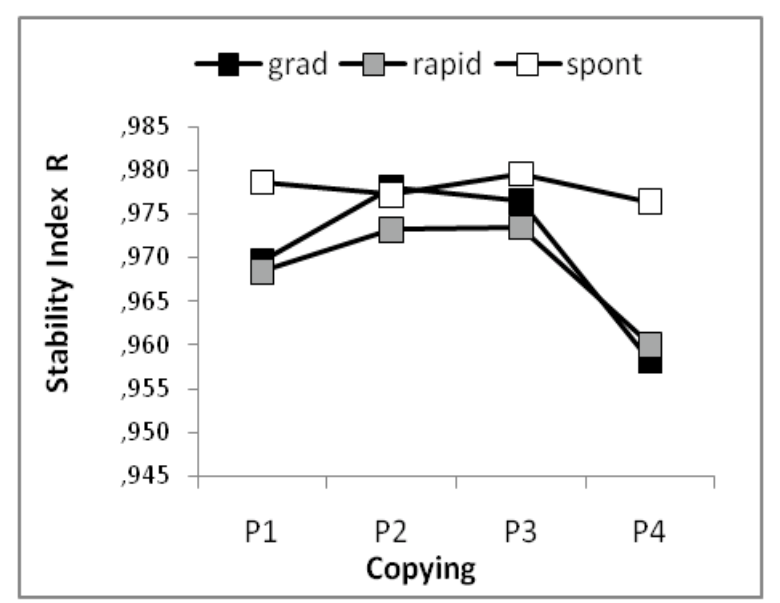

Fig. 3. Stability index $R$ as a function of copying Period and of Copying condition.

\section{Results}

\subsection{Number of cycles at copying}

A 4 (Period) $\times 3$ (Copying) ANOVA on the number of cycles revealed a main effect of Copying $(\mathrm{F}(2,22)=$ 20.939, $\left.\mathrm{p}<0.000, \eta^{2}=0.999\right)$, of Period $(\mathrm{F}(1.300$, $\left.14.298)=45.814, \mathrm{p}<0.000, \eta^{2}=0.999\right)$, and $\mathrm{a}$ significant Copying $\times$ Period interaction $(\mathrm{F}(1.931$, $\left.21.238)=27.475, p<0.000, \eta^{2}=0.999\right)$. For gradual Copying, 4 (Period) ANOVA revealed a main effect of Period $\left(\mathrm{F}(1.601,17.616)=10.085, \mathrm{p}<0.000, \eta^{2}=\right.$
0.996) ensuring us that participants decreased the number of cycles as a function of Period.

\subsection{Absolute Error at Copying}

Absolute Error is displayed in Figure 2 as a function of Pattern and of Period for the three copying conditions (left curves). A 3 (Copying) $\times 4$ (Period) $\times 5$ (Pattern) ANOVA on AE revealed a main effect of Copying $\left(\mathrm{F}(2,22)=3.989, \mathrm{p}<0.003, \eta^{2}=0.652\right)$, of Pattern $\left(F(2.620,28.816)=31.047, \mathrm{p}<0.000, \eta^{2}=\right.$ $0.999)$, and a significant Copying $\times$ Period $\times$ Pattern interaction $\left(F(2.685,29.535)=6.484, p<0.002, \eta^{2}=\right.$ $0.931)$. Subsequent t-tests indicated that, $\mathrm{AE}$ was lower for patterns produced at spontaneous than at rapid Copying $(\mathrm{t}(11)=3.119, \mathrm{p}<0.014 ; \mathrm{M}=4.531, \mathrm{SD}=$ 1.736 vs $\mathrm{M}=6.458, \mathrm{SD}=2,246$, respectively). For gradual Copying, polynomial contrasts indicated a significant quadratic trend as a function of Period $(\mathrm{F}(1$, $\left.11)=8.117, p<0.016, \eta^{2}=0.425\right)$, signaling that $\mathrm{AE}$ first decreased and then increased as a function of Period,. For rapid and spontaneous Copying, similar analysis exhibited a linear trend, indicating that $\mathrm{AE}$ decreased $\left(F(1,11)=6.618, p<0.026, \eta^{2}=0.376\right.$; $\mathrm{F}(1,11)=4.325, \mathrm{p}<0.045, \eta^{2}=0.174$, respectively).

\subsection{Stability Index at copying}

Stability Index is displayed in Figure 3 as a function of Pattern and of Period for the three copying conditions. A 3 (Copying) $\times 4$ (Period) $\times 5$ (Pattern) ANOVA on stability index at copying revealed a main effect of Copying $\left(\mathrm{F}(1.561,17.169)=5.042, \mathrm{p}<0.016, \eta^{2}=\right.$ $0.676)$, of Period $(\mathrm{F}(1.624,17.863)=4.121, \mathrm{p}<0.041$, $\left.\eta^{2}=0.598\right)$, of Pattern $(F(2.280,25.079)=18.963, p<$ $\left.0.000, \eta^{2}=0.999\right)$, and a significant Period $\times$ Pattern $\left(\mathrm{F}(2.625,28.875)=3.114, \mathrm{p}<0.047, \eta^{2}=0.623\right)$ and Copying $\times$ Period $\times$ Pattern $(\mathrm{F}(5.895,64.842)=$ $\left.2.241, \mathrm{p}<0.047, \eta^{2}=0.753\right)$ interactions. For gradual and rapid Copying, polynomial contrasts revealed a significant quadratic trend as a function of Period $\left(\mathrm{F}(1,11)=10.228, \mathrm{p}<0.008, \eta^{2}=0.483 ; \mathrm{F}(1,11)=\right.$ $6.878, p<0.024, \eta^{2}=0.385$, respectively), signaling that Stability Index first increased and then decreased over Periods in these conditions.

\subsection{Absolute error at reproduction}

A 3 (Copying) $\times 3$ (Interval) $\times 5$ (Pattern) ANOVA on AE revealed a main effect of Pattern $(F(4,44)=$ 12.109, $\left.\mathrm{p}<0.000, \eta^{2}=0.524\right)$, a Interval $\times$ Pattern interaction $\left(\mathrm{F}(8,88)=2.118, \mathrm{p}<0.036, \eta^{2}=0.524\right)$. 
AE was similar after spontaneous, rapid and gradual copying.

\subsection{Improvement Index}

Improvement Index is displayed in Figure 4 as a function of Copying condition and of Interval. A 3 (Copying) $\times 3$ (Interval) $\times 5$ (Pattern) ANOVA revealed a main effect of Copying $(\mathrm{F}(1.356,14.912)=$ 5.436, $\left.\mathrm{p}<0.026, \eta^{2}=0.663\right)$, of Pattern $(\mathrm{F}(2.240$, $\left.24.641)=3.302, p<0.049, \eta^{2}=0.602\right)$, a significant Copying $\times$ Pattern $(\mathrm{F}(4.041,44.453)=3.202, \mathrm{p}<$ $\left.0.019, \eta^{2}=0.798\right)$, Interval $\times$ Pattern $(\mathrm{F}(3.396$, $\left.37.353)=2.733, \mathrm{p}<0.049, \eta^{2}=0.658\right)$, and Copying $\times$ Interval $\times$ Pattern $(\mathrm{F}(6.316,369.477)=2.160, \mathrm{p}<$ $\left.0.018, \eta^{2}=0.928\right)$ interactions.

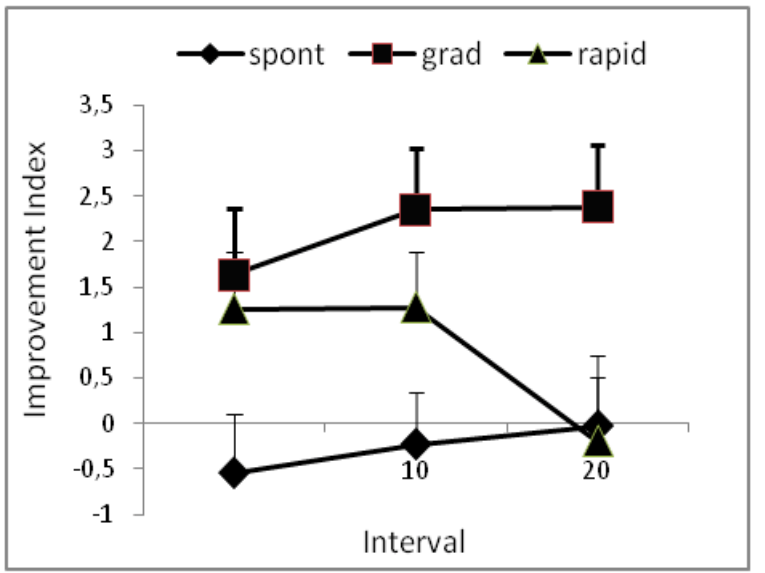

\section{Fig. 4. Improvement Index as a function of} Interval and Copying condition.

A subsequent 3 (Copying) $\times 5$ (Pattern) ANOVA carried out on Improvement Index for $0 \mathrm{sec}$ Interval indicated a main effect of Copying only $(\mathrm{F}(2,22)=$ $\left.3.733, p<0.040, \eta^{2}=0.621\right)$ : Improvement Index was higher for rapid than for spontaneous Copying condition $(\mathrm{t}(11)=2,827, \quad \mathrm{p}<0.025)$. The same analysis carried out for $10 \mathrm{sec}$ Interval revealed a main effect of Copying $\left(\mathrm{F}(2,22)=4.188, \mathrm{p}<0.029, \eta^{2}=\right.$ $0.675)$ and of Pattern $(\mathrm{F}(2.730,30.026)=3.155, \mathrm{p}<$ $\left.0.043, \eta^{2}=0.646\right)$ : Improvement Index was higher for gradual than for spontaneous Copying $(\mathrm{t}(11)=2.514, \mathrm{p}$ $<0.043)$. For $20 \mathrm{sec}$ Interval, the same analysis exhibited a main effect of Copying $(\mathrm{F}(2,22)=6.843$, $\mathrm{p}$ $\left.<0.005, \eta^{2}=0.752\right)$, and a significant Copying $\times$ Pattern interaction $(\mathrm{F}(3.952,43.470)=4.059, \mathrm{p}<$ $\left.0.007, \eta^{2}=0.625\right)$ : Improvement Index was higher for gradual than for spontaneous Copying $(\mathrm{t}(11)=3.396, \mathrm{p}$ $<0.008)$ and higher for gradual than for rapid Copying $(\mathrm{t}(11)=3.359, \mathrm{p}<0.009)$.

\section{Conclusion}

Stable graphic patters were destabilized at copying and the evolution of this destabilization effect was distinct in spontaneous, rapid and gradual condition. The required graphic patterns were less accurate and stable when they were traced at rapid rather than at spontaneous movement frequency. For gradual copying, accuracy and stability dropped between the third and last copying period.

The destabilization effect was forgotten: accuracy improved from copying to reproduction. Although overall accuracy during the reproduction phase was similar for all the copying conditions, the forgetting of the destabilization effect was sensitive to how required movement frequency changed at copying. After an abrupt change from the rapid to the spontaneous movement frequency during copying, an improvement in accuracy appeared rapidly, for the $0 \mathrm{~s}$ reproduction interval. After a gradual decrease in movement frequency, an improvement in accuracy arose later, for the 10 and $20 \mathrm{~s}$ interval. These findings are in line with the claim of Huang and Shadmehr [1] suggesting that the evolution of forgetting depends on the evolution of environmental requirements at encoding (cf. copying): abrupt changes of environmental requirements at encoding lead to rapid changes in performance at recall (cf. reproduction) and gradual changes at encoding to slow changes at recall.

Comparison of performance at copying and reproduction revealed that concomitant, abrupt degradation in accuracy and stability at copying led to late improvement at reproduction. Abrupt degradation in stability at copying led to immediate improvement.

\section{References}

[1] V.S. Huang and R., Shadmehr, Persistence of motor memories reflects statistics of the learning event, Journal of Neurophysiology, 102, 931-940, 2009.

[2] J.A.S. Kelso (1984). Phase transitions and critical behavior in human bimanual coordination. American Journal of Physiology, Regulatory, Integrative and Comparative Physiology, 15, R1000-R1004, 1984.

[3] H. Haken, J.A.S. Kelso, and H. Buntz, A theoretical model of phase. Transitions in human movements. Biological Cybernetics, 51, 347-356, 1985.

[4] S. Athènes, I. Sallagoity, P.G. Zanone, and J. M. Albaret, (2004). Evaluating the coordination dynamics of handwriting. Human Movement Science, 23(5), 621641, 2004.

[5] I. Sallagoïty, S. Athènes, P.G. Zanone, and J. M Albaret, Stability of coordination patterns in handwriting: effects of speed and hand. Motor Control, 8(4), 405-421, 2004. 